Case

Report

\title{
A Rare Case of Multiple Myxoma Involving Both Mitral Valve Leaflets
}

\author{
Xiao-Dong Li, MD, ${ }^{1}$ Yang Bai, $\mathrm{PhD},{ }^{2}$ Xiu-Mei Duan, $\mathrm{PhD},{ }^{3}$ \\ and Xiao-Cong Wang, $\mathrm{MD}^{1}$
}

\begin{abstract}
Mitral valve myxoma is a very rare entity. Multiple myxomas with extensive involvement of the anterior and posterior leaflets of the mitral valve are exceedingly rare. We report a 58-year-old man who was admitted as sudden syncope. Thoracic echocardiography showed several masses adherent to the anterior and posterior leaflets and the mitral annulus with obvious mobility. Intraoperative probing revealed multiple tumors attached to the mitral annulus, valve leaflets, and tendinous cords. Mechanical mitral valve replacement was performed. Histopathological examination confirmed all tumors to be myxomas.
\end{abstract}

Keywords: cardiac tumor, myxoma, mitral valve, echocardiography

\section{Introduction}

Cardiac myxoma is one of the most common primary cardiac tumors, majority of which occur in the left atrium. ${ }^{1-3)}$ Cardiac myxoma can occur at any age; the incidence is higher among women. ${ }^{1-3)}$ Mitral valve myxoma is a very rare clinical entity; only a few cases have been reported till date from across the world. $\left.{ }^{4}\right)$ Multiple myxomas with extensive involvement of the anterior and posterior mitral leaflets and chordae tendineae are exceedingly rare. We herein report a 58-year-old man with multiple myxomas adhered to the anterior and posterior leaflets and the anterior mitral annulus.

${ }^{1}$ Department of Echocardiography, Cardiovascular Disease Center, The First Hospital of Jilin University, Changchun, Jilin China ${ }^{2}$ Department of Cardiac Surgery, The First Hospital of Jilin University, Changchun, Jilin, China

${ }^{3}$ Department of Pathology, The First Hospital of Jilin University, Changchun, Jilin, China

Received: October 1, 2017; Accepted: December 8, 2017 Corresponding author: Xiao-Cong Wang, MD. Department of Echocardiography, Cardiovascular Disease Center, The First Hospital of Jilin University, Changchun, Jilin 130021, China

Email: wangxiaocong419@163.com

(C)2019 The Editorial Committee of Annals of Thoracic and Cardiovascular Surgery. All rights reserved.

\section{Case Report}

A 58-year-old man was referred to our hospital with sudden syncope as the main symptom. His body temperature, respiration, blood pressure, and pulse were normal. His past medical history was unremarkable. There was no recent history of change in body weight. On auscultation, systolic murmurs at the cardiac apex were detected. Electrocardiogram (ECG) showed normal sinus rhythm.

Thoracic echocardiography (apical four-chamber view, parasternal, and apical long axis views) of the left heart showed several hyperechogenic masses adhered to the anterior and posterior leaflets of the mitral valve and the anterior mitral annulus. The neoplasms measured $20 \times 13 \mathrm{~mm}, 21 \times 12 \mathrm{~mm}$ and $6 \times 3 \mathrm{~mm}$, respectively. These loose-structured tumors showed broad-based attachment with the mitral valve, showed obvious mobility during the cardiac cycle, and showed a certain degree of deformability. The neoplasms remained in the left atrium during systole, and prolapsed into the left ventricle during diastole, which caused a slight increase in the velocity of blood flow at the mitral orifice during diastole. Doppler examination showed a maximum flow velocity of $130 \mathrm{~cm} / \mathrm{s}$; color Doppler flow imaging (CDFI) displayed slight-to-moderate regurgitation signal at the 
mitral orifice (Fig. 1). These tumors were provisionally diagnosed as mitral valve myxomas based on ultrasound findings; however, mitral valve vegetations could not be excluded.

The patient accepted open cardiac surgery for neoplasm excision under general anesthesia and extracorporeal circulatory support. Intraoperative probing revealed multiple myxoid tumors attached to the mitral annulus, valve leaflets, and tendinous cords, both at the left atrium and the left ventricle. The mitral valve was removed to ensure complete resection of the tumors. The mitral leaflets and part of mitral annulus, valve leaflets, and tendinous cords adherent to the neoplasms were also removed, and Sorin Bicarbon mechanical mitral valve replacement was performed. Histopathological examination of surgical specimen confirmed the neoplasms to be myxomas (Fig. 2). Postoperatively, the patient was treated with heparin combined with warfarin anticoagulation and followed-up for 1 year. There were no signs of recurrence on follow-up transthoracic echocardiography; the function of mechanical valve was good.

\section{Discussion}

Cardiac tumors can be divided into primary and secondary tumors; the majority of primary cardiac tumors are benign. ${ }^{1-3)}$ Myxoma is the most common primary cardiac tumor, which accounts for $30 \%-50 \%$ of all cardiac tumors. ${ }^{1)}$ Most myxomas occur in the left atrium, followed by the right atrium, left ventricle, and right ventricle, respectively. ${ }^{3)}$ Myxomas typically occur as a single mass; multiple myxomas are rare. ${ }^{5,6)}$ Myxoma occurs at the cardiac valves is rare, ${ }^{4)}$ while myxoma that simultaneously involves the anterior and posterior valves of mitral valve is even rarer. The myxoma of our patient simultaneously involved the anterior and posterior leaflets and chordae tendineae of mitral valve, which is definitely scarce.

Mitral valve myxoma should mainly be differentiated from mitral valve vegetations, papillary fibroelastoma, and thrombus. ${ }^{1)}$ Mitral valve vegetations often occur in patients with infective endocarditis; these are inflammatory lesions caused by bacterial or other infections of the heart valve. ${ }^{7)}$ Mitral valve vegetations are often attached to the left atrium, and appear as masses or flakes of different sizes. Larger vegetations typically show high mobility during the cardiac cycle. The echo intensity of mitral valve vegetations increases with progression of the disease course, and the valve leaflets develop irregular thickening. The majority of papillary fibroelastomas occur at

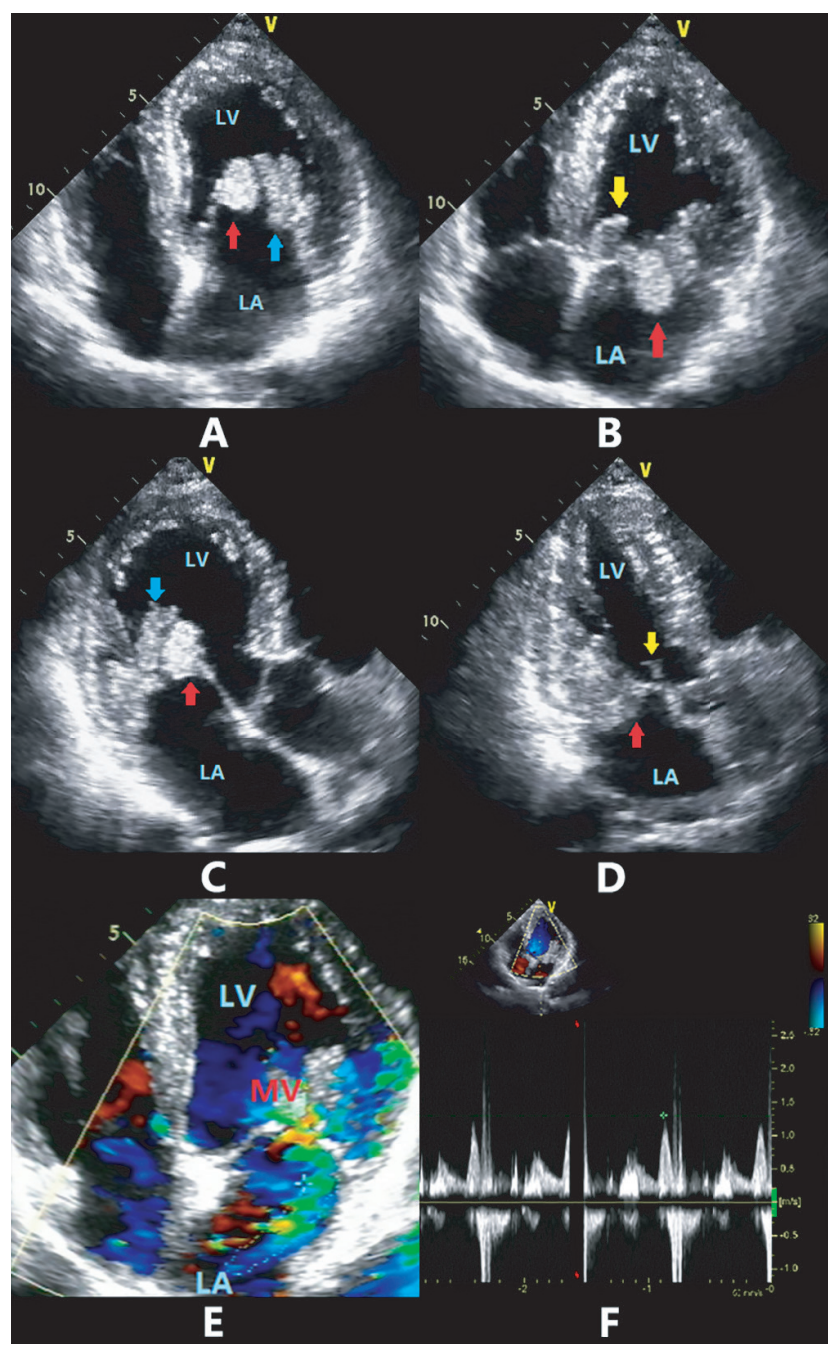

Fig. 1 Echocardiography images. The diastolic period of both apical four-chamber view (A) and apical long axis views $(\mathbf{C})$ showed two tumor masses $(20 \times 13 \mathrm{~mm}$ [red arrows] and $21 \times 12 \mathrm{~mm}$ [blue arrow]) adherent to the anterior and posterior mitral valves. The loose-structured masses have a broad-based attachment with the mitral valves, and show large degree of mobility during the cardiac cycle and a certain degree of deformation. The masses are located in the left atrium during systole (B and D) and prolapsed into the left ventricle (A and C) through the mitral valve opening during diastole. Both apex four-chamber view (B) and the apical long axis view (D) during systole show a smaller substantive tumor $(8 \times 3 \mathrm{~mm})$ at the left ventricular side of the root of the anterior mitral valve (yellow arrow). The blood flow velocity through the mitral valve during diastole is slightly faster (maximum flow velocity: $130 \mathrm{~cm} / \mathrm{s}$ ) (F). CDFI revealed a slight-to-moderate regurgitation signal at the mitral valve (E). LA: left atrium; LV: left ventricular; MV: mitral valve; CDFI: color Doppler flow imaging 


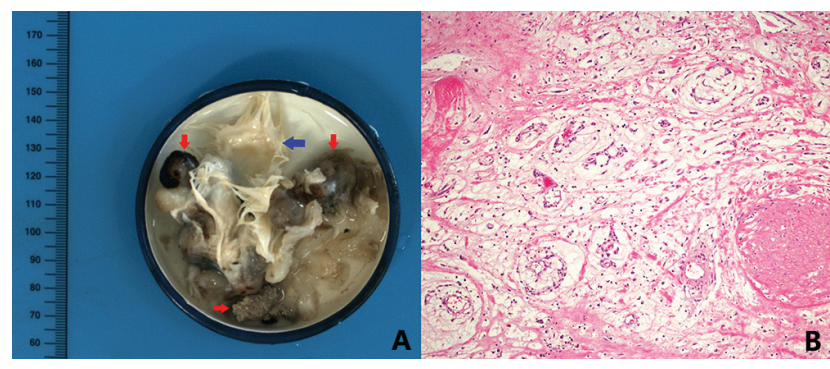

Fig. 2 Histopathological examination of sections of lesions. The gross specimen (A) shows light brown, gray, and brown tissues, with a solid, soft, and translucent cross section (red arrows), which were attached to the mitral valve (blue arrow). The microscopic image (B) confirmed the masses to be myxomas (HE staining, $\times 100$ magnification).

the valves, most commonly at the aortic and mitral valves; the tricuspid and pulmonary valves, atrium and ventricle are uncommon sites. ${ }^{8,9)}$ Papillary fibroelastoma usually appears as a small well-delineated mass at valve with uniform echogenicity on two-dimensional (2D) echocardiography. The shape of papillary fibroelastoma may be oval, circular, or irregular; these are usually located at the atrial surface of the atrioventricular valves and at the ventricular surface of the arterial valves. ${ }^{8)}$ Mitral valve thrombosis is a rare entity, and typically occurs after mitral valve replacement. ${ }^{10)}$ Postoperative thrombosis of mitral valve is a severe complication of mechanical valve replacement surgery. ${ }^{10)}$ The more active thrombosis with pedicle is very rare. ${ }^{11)}$ Most thrombi are less mobile, lack a pedicle, and the shape generally does not change during the cardiac cycle, which differentiates it from myxoma. ${ }^{11}$

Thoracic echocardiography is the investigation of choice for detection of heart valve disease because it is simple, convenient, and easily manipulated. ${ }^{1)}$ The mitral valve myxomas in the present case were multiple and showed obvious mobility, due to which they were likely to be misdiagnosed as vegetations. As this patient had no history of fever, mitral valve myxoma was a distinct possibility. Diagnosis was confirmed with histopathology eventually. Furthermore, the myxomas had widely involved the mitral valve annulus, valve leaflets, and chordae tendineae tissue, and could not be completely removed; therefore, the mechanical valve replacement was performed for this patient. The postoperative follow-up mainly focused on the function of the mechanical valve and whether there is thrombosis; we also observed for the recurrence of cardiac myxoma. Our patient was followed-up for 1 year with ultrasound, and the function of mechanical valve remained good without recurrence of cardiacmyxoma.

\section{Author Contributions}

Li Xiao-Dong contributed to research design, data acquisition and analysis, drafting article, and the approval of the submitted and final versions.

Bai Yang contributed to data acquisition and analysis, and the approval of the submitted and final versions.

Duan Xiu-Mei contributed to data collection and the approval of the submitted and final versions.

Wang Xiao-Cong contributed to research concept, critical revision of article, and completing the approval of the submitted and final versions.

\section{Disclosure Statement}

The authors declare that they have no conflicts of interest.

\section{References}

1) $\mathrm{Yu} \mathrm{L}, \mathrm{Gu} \mathrm{T}$, Shi E. Echocardiographic findings and clinical correlation with cardiac myxoma. JACC Cardiovasc Imaging 2016; 9: 618-21.

2) Pergolini A, Zampi G, Tinti MD, et al. The strange case of the infarcted myxoma. Echocardiography 2016; 33: 476-8.

3) Lin Y, Xiao J, Chen J, et al. Treating cardiac myxomas: a 16-year Chinese single-center study. J Cardiovasc Med (Hagerstown) 2016; 17: 44-53.

4) Nya F, Abdou A, Bamous M, et al. [Myxoma involving posterolateral leaflet: about a case]. Pan Afr Med J 2017; 26: 61. (in French)

5) Schmidt C, Doi A, Ura M, et al. Familial atrial myxoma: three related cases at an Australian tertiary institution. Ann Thorac Cardiovasc Surg 2017; 23: 203-6.

6) Kataoka S, Otsuka M, Goto M, et al. Primary multiple cardiac myxomas in a patient without the carney complex. J Cardiovasc Ultrasound 2016; 24: 71-4.

7) Cahill TJ, Prendergast BD. Infective endocarditis. Lancet 2016; 387: 882-93.

8) Ryu SW, Beom MS, Kim SR, et al. Cardiac papillary fibroelastoma. Rev Port Cardiol 2015; 34: 507-8.

9) Kim M, Kim SH, Moon SY, et al. Native aortic valve thrombosis resembling papillary fibroelastoma. J Cardiovasc Ultrasound 2014; 22: 148-50.

10) Mehra S, Movahed A, Espinoza C, et al. Horseshoe thrombus in a patient with mechanical prosthetic mitral valve: a case report and review of literature. World J Clin Cases 2015; 3: 838-42.

11) Li X, Chen L, Duan X, et al. A rare case of a pedicled mobile thrombus in right atrium. Heart Surg Forum 2016; 19: E269-E271. 